\title{
Single Pulse Laser Ablation of AISI 316L Stainless Steel Surface Using Nd:YAG Laser Irradiation
}

\author{
P. DEMIR ${ }^{a, b, *}$, E. KACAR ${ }^{a, b}$,E. AKMAN ${ }^{b}$ AND A. DEMIR ${ }^{b, c}$ \\ ${ }^{a}$ Kocaeli University, Faculty of Arts and Science, Department of Physics, 41380, Kocaeli, Turkey \\ ${ }^{b}$ Kocaeli University, Laser Technologies Research and Application Center, 41275, Kocaeli, Turkey \\ ${ }^{c}$ Kocaeli University, Electro Optics System Engineering, 41380, Kocaeli, Turkey
}

The interaction of single-pulse Nd:YAG laser, operating at $1064 \mathrm{~nm}$ wavelength and $6 \mathrm{~ns}$ pulse duration, with AISI 316L stainless steel target surface was investigated experimentally and theoretically. Surface modification of stainless steel using laser irradiation was studied by observing the effects of varying incident laser pulse intensities on surface morphology. Surface structure of laser treated stainless steel was determined by optical microscopy and profilometry analyses. Numerical calculation by heat transfer equation was performed for single laser pulse irradiation. The results, obtained by theoretical and experimental processes, of the interaction between single-pulse Nd:YAG laser irradiation and AISI 316L stainless steel target surface are reported.

DOI: $10.12693 /$ APhysPolA.125.439

PACS: 42.62.-b, 44.05.+e, 44.10.+i, 61.82.-d, 61.82.Bg, 79.20.Eb

\section{Introduction}

Removing material from a target surface by irradiation with a laser beam is the process of laser ablation. There are several applications based on laser ablation, such as surface modification of materials [1], chemical analysis [2], micromachining [3] and thin film deposition [4]. Since the process depends on the optical and thermal properties of the target [5] and the laser parameters [6], it is necessary to investigate the effects of the parameters and energy transfer to the target material in order to control and optimize the laser-induced material removal process $[7,8]$.

In this study, the interaction of Nd:YAG laser beam of $1064 \mathrm{~nm}$ wavelength with AISI 316L stainless steel target surface was investigated experimentally and theoretically.

\section{Experimental}

AISI 316L stainless steel was used as a target material. Elemental composition of the material comprises 69 wt $\%$ Fe, 18 wt\% Cr, 10 wt\% Ni, 3 wt\% Mo. The stainless steel plate has the thickness of $1 \mathrm{~mm}$ and a surface roughness less than $1 \mu \mathrm{m}$. Prior to laser treatment, the polished surface of steel plate was cleaned with acetone and ethanol.

The experiments were carried out using Nd:YAG Surelite Continuum laser system generating pulses of $6 \mathrm{~ns}$ duration. Single-pulse laser irradiation was applied onto the surface of the target material. The fundamental Nd:YAG wavelength of $1064 \mathrm{~nm}$ was used for processing. During

*corresponding author; e-mail: pinardemir@kocaeli.edu.tr the irradiation process the laser system was operated in the fundamental spatial mode, and the spatial intensity distribution of the laser beam is characterized by Gaussian profile. Diameter of the laser beam was $9.8 \mathrm{~mm}$. An iris was used as a mask to shape the laser beam. Mask aperture has been used in a few studies to shape the laser beam $[9,10]$. The laser beam was focused by a $100 \mathrm{~mm}$ focal length lens to the surface of the stainless steel material at normal direction. The experiments were performed in air. Intensity of the incident laser pulse on the target material surface was varied by changing the distance between lens and target at fixed laser pulse energy.

\section{Numerical calculation}

The transient temperature distribution in the target material is obtained using the Fourier heating model by solving one-dimensional heat conduction equation since the heat transfer calculations is performed for nanosecond laser pulse, and the absorption length in the target is much smaller than the laser beam diameter [11-14]. The heat conduction equation for laser heating pulse with a Gaussian intensity profile can be written as

$$
\rho c_{p} \frac{\partial T(x, t)}{\partial t}=\frac{\partial}{\partial x}\left(\kappa \frac{\partial T(x, t)}{\partial x}\right)+S_{0},
$$

where $\rho$ is the mass density, $c_{p}$ is the specific heat, $\kappa$ is the thermal conductivity, $T$ is the temperature inside the target material, $t$ is the time, $x$ is the position from the material surface. All the thermal properties of the target material are assumed to be constant. $S_{0}$ is the energy source term and denotes heat generation by laser energy absorption

$$
S_{0}=\alpha(1-R) I_{0}(t) \exp (-\alpha x),
$$

where $R$ and $\alpha$ are the surface reflectivity and the absorption coefficient of the material, respectively. $I_{0}(t)$ is 
the incident laser irradiance at the surface, and can be written as

$$
I_{0}(t)=I_{0} \exp \left((-4 \ln 2)\left(\frac{t}{t_{p}}-1.5\right)^{2}\right) .
$$

Here $t$ is time and $t_{p}$ is the temporal full-width at half-maximum.

When the target material is irradiated by laser pulse, temperature at the target surface rises. During the heating process, when the temperature reaches the melting temperature of the target, material starts to melt. If enough energy is supplied, subsequent evaporation of material occurs. The local temperature remains constant during phase transition process. Phase change process was taken in consideration as in the model explained by Yilbas et al. [15]:

$$
\begin{aligned}
& \rho_{m} L_{m} \frac{\partial x_{m}}{\partial t}=\frac{\partial}{\partial x}\left(k_{m} \frac{\partial T}{\partial x}\right)+S_{0}, \\
& \rho_{b} L_{b} \frac{\partial x_{b}}{\partial t}=\frac{\partial}{\partial x}\left(k_{b} \frac{\partial T}{\partial x}\right)+S_{0} .
\end{aligned}
$$

Governing equations are solved numerically using the finite difference method. Explicit scheme is applied for discretization of temporal domain. However implicit scheme is also applied to compare the solutions obtained from that of explicit scheme. Explicit scheme solution is used since the solutions are the same. All the thermal properties are assumed to be constant in simulations.

\section{Results}

Theoretical analysis is performed to determine the processes of heating, melting, and ablation of steel material in order to get an understanding of physical processes occurring during the laser ablation. A parametric study is performed to determine the ablation threshold for different laser fluences (Fig. 1a,b). The damage threshold is defined as the minimum laser radiation energy density required for creating detectable damage on the target [16]. It is observed from results that the damage threshold for the Nd:YAG (1064 nm)-stainless steel interaction was about $2 \mathrm{~J} / \mathrm{cm}^{2}$.

In the experiment, laser pulse energy was set at the value of $20 \mathrm{~mJ}$. The pulse energy was determined by a powermeter. The distance between the focusing lens and the target was adjusted from $100 \mathrm{~mm}$ to $90 \mathrm{~mm}$. Correspondingly, the laser beam spot size on the target surface varied between 43 and $316 \mu \mathrm{m}$ in diameter, and the peak intensity of the laser beam was in the range $4.25 \times 10^{9}$ $2.27 \times 10^{11} \mathrm{~W} / \mathrm{cm}^{2}$. In the other series of experiments, for each lens-target distance we fixed the laser pulse energy to $50 \mathrm{~mJ}$, or $200 \mathrm{~mJ}$ by changing the laser lamp current. Correspondingly, the intensity in the laser beam spot was in the range $1.06 \times 10^{10}-5.67 \times 10^{11} \mathrm{~W} / \mathrm{cm}^{2}$, or $4.25 \times 10^{10}-2.27 \times 10^{12} \mathrm{~W} / \mathrm{cm}^{2}$. Surface structure of laser treated stainless steel was determined by optical microscopy and profilometry analyses. Melt depth and vapour depth is calculated for energies used in ex-
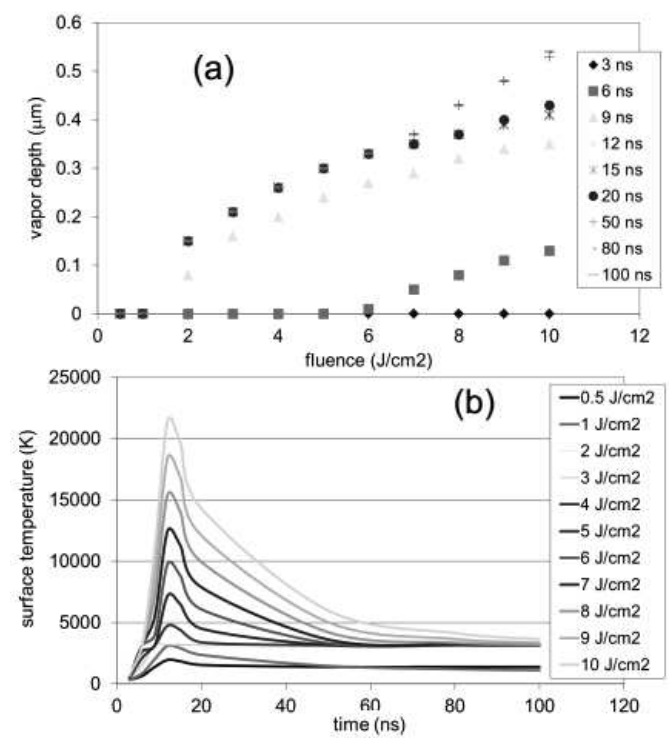

Fig. 1. (a) Vapor depth and (b) surface temperature of steel target for different laser fluences.

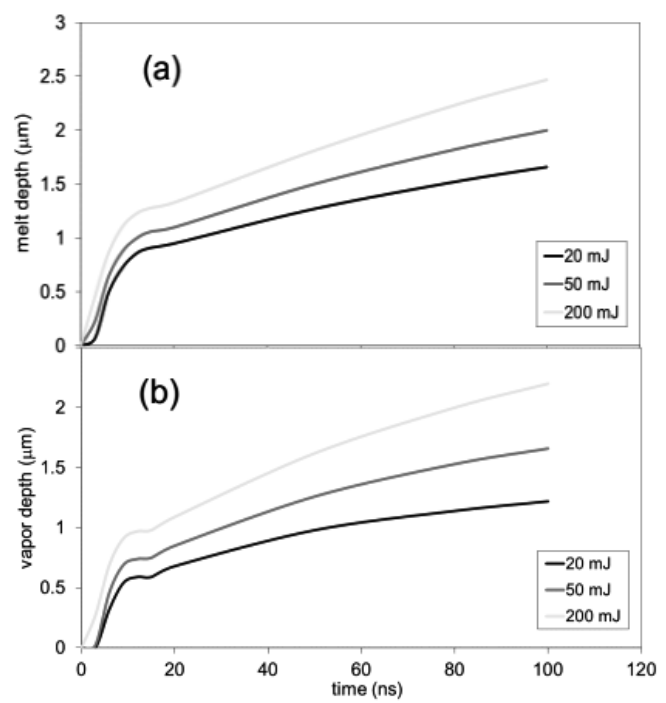

Fig. 2. Transient (a) melt depth and (b) vapor depth of steel target for different laser pulse energies used in experiment.

periment. Transient melt and vapor depth profiles are shown in Fig. 2a,b.

The surface morphology of the stainless steel material obtained by optical microscope and profilometer is shown in Fig. 3 and Fig. 4, respectively. It can be observed from Fig. 4a that there is wave-like movement in the liquid phase of the material during processing. It can be revealed from Fig. $4 \mathrm{~b}$ that the surface oxidation can occur since the increase in the volume of the laser-treated material is observed. Similar observations on titanium material were reported by György et al. [17]. 


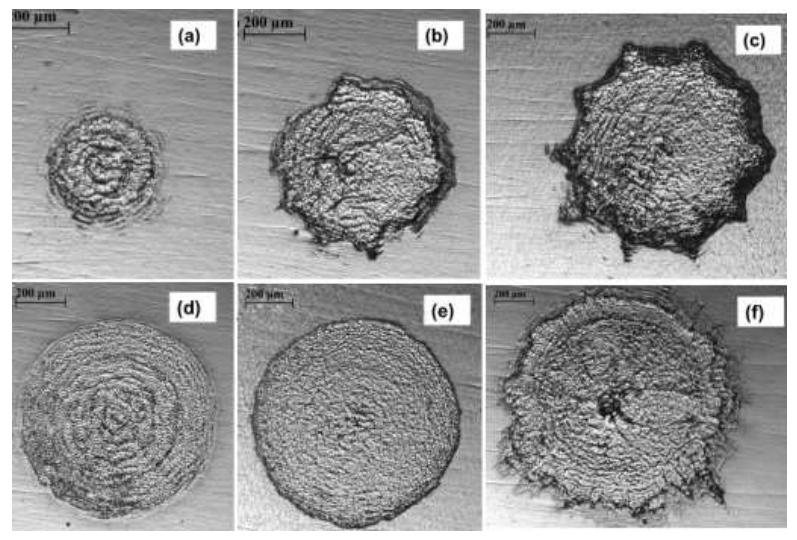

Fig. 3. Optical microscope images for (a), (d) $20 \mathrm{~mJ}$, (b), (e) $50 \mathrm{~mJ}$, (c), (f) $200 \mathrm{~mJ}$ energies at lens-target distance (a), (b), (c) $100 \mathrm{~mm}$, and (d), (e), (f) $90 \mathrm{~mm}$.

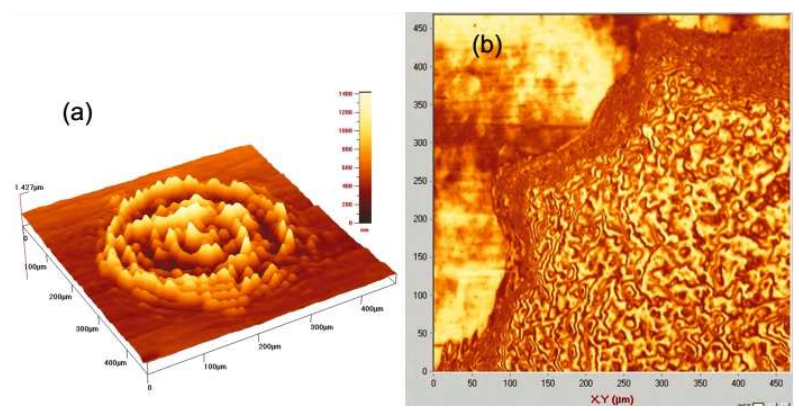

Fig. 4. Profilometer images for (a) $20 \mathrm{~mJ}$ energy for lens-target distance $100 \mathrm{~mm}$, (b) for $200 \mathrm{~mJ}$ energy for lens-target distance $100 \mathrm{~mm}$.

\section{Conclusion}

The interaction of single pulse Nd:YAG laser, operating at $1064 \mathrm{~nm}$ wavelength, with AISI 316L stainless steel target surface was investigated experimentally and theoretically. Surface modification of stainless steel by laser was studied by changing incident laser pulse intensity. The effects of different laser intensities on surface morphology were studied.

\section{Acknowledgments}

This study was supported by Scientific Research Projects Unit of Kocaeli University, project No. 2010/ 072.

\section{References}

[1] H. Li, S. Costil, V. Barnier, R. Oltra, O. Heintz, C. Coddet, Surf. Coat. Technol. 201, 1383 (2006).

[2] M.A. Shannon, X.L. Mao, A. Fernandez, W.-T. Chan, R.E. Russo, Anal. Chem. 67, 4522 (1995).

[3] L. Tunna, A. Kearns, W. O'Neill, C.J. Sutcliffe, Opt. Laser Technol. 33, 135 (2001).

[4] S. Weißmantel, G. Reiße, Appl. Surf. Sci. 197-198, 331 (2002).

[5] D. Bleiner, Z.Y. Chen, D. Autrique, A. Bogaerts, J. Anal. At. Spectrom. 21, 910 (2006).

[6] X. Zeng, X.L. Mao, R. Greif, R.E. Russo, Appl. Phys. A, Mater. Sci. Process. 80, 237 (2005); B. Zhang, K.C. Yung, Opt. Laser Eng. 44, 815 (2006).

[7] E. Amer, P. Gren, A.F.H. Kaplan, M. Sjödahl, M. El Shaer, Appl. Surf. Sci. 256, 4633 (2010).

[8] D.A. Willis, X. Xu, Int. J. Heat Mass Transf. 45 , 3911 (2002).

[9] J. Greuters, N.H. Rizvi, Proc. SPIE 4941, 77 (2003).

[10] N.H. Rizvi, Proc. SPIE 3680, 546 (1999).

[11] B.S. Yilbas, S.Z. Shuja, A. Arif, M.A. Gondal, J. Mater. Process. Technol. 135, 6 (2003).

[12] A. Bogaerts, Z. Chen, R. Gijbels, A. Vertes, Spectrochim. Acta B, At. Spectrosc. 58, 1867 (2003).

[13] J.R. Ho, C.P. Grigoropoulos, J.A.C. Humphrey, J. Appl. Phys. 78, 4696 (1995).

[14] W. Svendsen, O. Ellegaard, J. Schou, Appl. Phys. A, Mater. Sci. Process 63, 247 (1996).

[15] B.S. Yilbas, S.B. Mansoor, R. Mansoor, Opt. Laser Technol. 40, 723 (2008).

[16] M. Trtica, B. Gakovic, D. Batani, T. Desai, P. Panjan, B. Radak, Appl. Surf. Sci. 253, 2551 (2006).

[17] E. György, I.N. Mihailescu, P. Serra, A. Pérez del Pino, J.L. Morenza, Surf. Coat. Technol. 154, 63 (2002). 